\title{
Pneumatosis Intestinalis and Portal Venous Gas: An Unusual Complication of Fecal Impaction
}

\author{
Abdo Zeinoun ${ }^{\mathrm{a}, \mathrm{b}}$, Clive Persaud ${ }^{\mathrm{a}}$, Jamshed Zuberi ${ }^{\mathrm{a}}$
}

\begin{abstract}
We present an unusual case of pneumatosis intestinalis and portal venous gas due to massive fecal impaction resulting in ischemic infarction of three separate bowel segments. The patient was taken emergently to the operating room for an exploratory laparotomy with resection and anastomosis of a proximal loop of the jejunum, distal loop of the jejunum, and most of the terminal ileum, with creation of a loop ileostomy. In our review of the literature, there have been only two similar cases of pneumatosis intestinalis/portal venous gas caused by fecal impaction requiring operative intervention.
\end{abstract}

Keywords: Pneumatosis intestinalis; Portal venous gas; Mesenteric ischemia; Loop ostomy

\section{Introduction}

Pneumatosis intestinalis (PI) and portal venous gas (PVG) are radiological signs of an intra-abdominal pathology that may be associated with mechanical obstruction, mesenteric ischemia, or idiopathic causes [1]. PI refers to gas circulating in the luminal wall of the gastrointestinal (GI) system such as the stomach, small or large bowel [1]. PVG refers to gas which has leaked from the GI system into the portal venous system, such as the portal vein [1]. We present a rare case of PI with PVG in an adult with dementia secondary to traumatic brain injury (TBI) that was treated with surgical resection and anastomosis of three portions of the small bowel.

\section{Case Report}

This is a 48-year-old male with a past medical history of de-

Manuscript submitted November 22, 2019, accepted December 9, 2019

aSt. Joseph's Regional Medical Center in Paterson, 703 Main St., Paterson, NJ 07503, USA

bCorresponding Author: Abdo Zeinoun, St. Joseph's Regional Medical Center in Paterson, 703 Main St., Paterson, NJ 07503, USA.

Email: azeinoun@sgu.edu

doi: https://doi.org/10.14740/jcs398 mentia secondary to TBI, hypertension (HTN), stage IV sacral decubitus ulcer and nonverbal at baseline with past surgical history of gastrostomy tube, who presented to the emergency department from a nursing home after being found to be afebrile with one episode of emesis and abdominal distension. Approximately 1 day prior to presentation, the patient pulled out the gastrostomy tube and a foley catheter was inserted in its place at an outside facility.

\section{Investigations}

A computed tomography (CT) of the abdomen/pelvis demonstrated extensive pneumatosis of the small bowel with portal and intra-hepatic gas and extensive fecal impaction (Figs. 1, 2). Intraoperatively, there were gangrenous parts of the jejunum and ileum along with a hugely distended large bowel due to fecal impaction (Fig. 3). White blood cell (WBC) count was $25.9 \times 10^{3} / \mu \mathrm{L}$, and lactate level of 9.7 $\mathrm{mmol} / \mathrm{L}$.

\section{Differential diagnosis}

Upon presentation and examination, PI with PVG, sepsis, and constipation with fecal impaction were leading differentials. Upon further inspection in the operating room a diagnosis of mesenteric ischemia was made.

\section{Treatment}

An emergency exploratory laparotomy was performed. A resection and anastomosis were performed to the proximal loop of the jejunum, distal loop of the jejunum, and most of the terminal ileum except for the distal most portion allowing formation of subsequent loop ostomy.

\section{Outcome and follow-up}

Ultimately, approximately $70 \mathrm{~cm}$ was left of small bowel including the duodenum. On postoperative day 2 of our case, total parenteral nutrition (TPN) was started to assess bowel function as there was concern for short bowel syndrome. The patient tolerated tube feeds and the ileostomy was viable. 

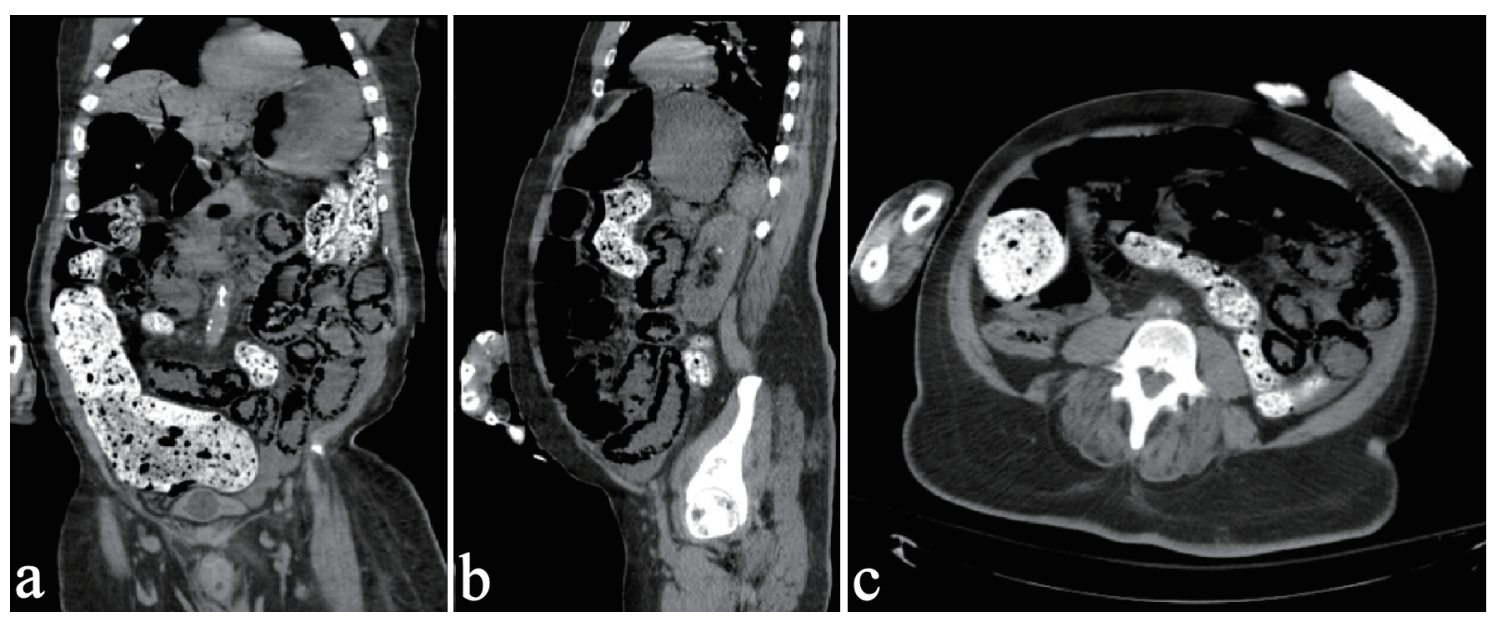

Figure 1. Pneumatosis intestinalis within the small bowel on frontal (a), sagittal (b) and transverse (c) plane on CT abdomen and pelvis, with fecal impaction in the large intestines. CT: computed tomography.

\section{Discussion}

Three general clinical etiologies have been postulated to cause PI with or without PVG: 1) Mechanical disease; 2) Mesenteric ischemia; and 3) Benign idiopathic [1]. Of those etiologies, stercoral perforation is the most life-threatening, and represents $3.2 \%$ of all colonic perforations [2]. This is an unusual cause of PI and/or PVG as the mechanism is due to a formation of a fecaloma large enough to cause distension of the bowel with a significant increase in pressure causing a perforation $[2,3]$. Our 48 -year-old male in this study presented without a fever, one episode of emesis and abdominal distension. The patient did not have an abdominal wall hernia or adhesions but did present with massive fecal impaction on radiology, that was causing significant bowel obstruction and back up in the large intestines, but ultimately the etiology is unknown. Two potential etiologies in this case are mesenteric ischemia or stercoral fecaloma that may have led to significant increased intraluminal pressure in the bowel. Of note, a presentation like this has only briefly been described in our review of the literature.

\section{Learning points}

Learning points from this case are: 1) PI and PVG are radiographic signs that point to an intra-abdominal pathology most often related to ischemia secondary to multiple primary pathologies [1]. 2) A lactic acid level $>2.0 \mathrm{mmol} / \mathrm{L}$ with $\mathrm{PVG}$ should warrant consideration for surgical decompression, thus correlation of lactic acid level with clinical presentation will help guide the type of therapy needed. 3) Early surgical decompression, resection and exteriorization, if indicated, are critical in post-diagnosis recovery, and decrease mortality rather than admission to the intensive care unit [1]. It is better judgement to take the patient to the operating room for exploration, admitted to the surgical intensive care unit, and then undergo tertiary wound closure to allow for re-exploration the next day. 4) Though mesenteric ischemia is a potential etiology, depending on the degree of severity such as extensive infarction resulting in black and necrotic bowel, surgical treatment may be futile. The best course of action in such cases is the least invasive option in accordance with the patient's/family's last wishes. 5) Short bowel syndrome is
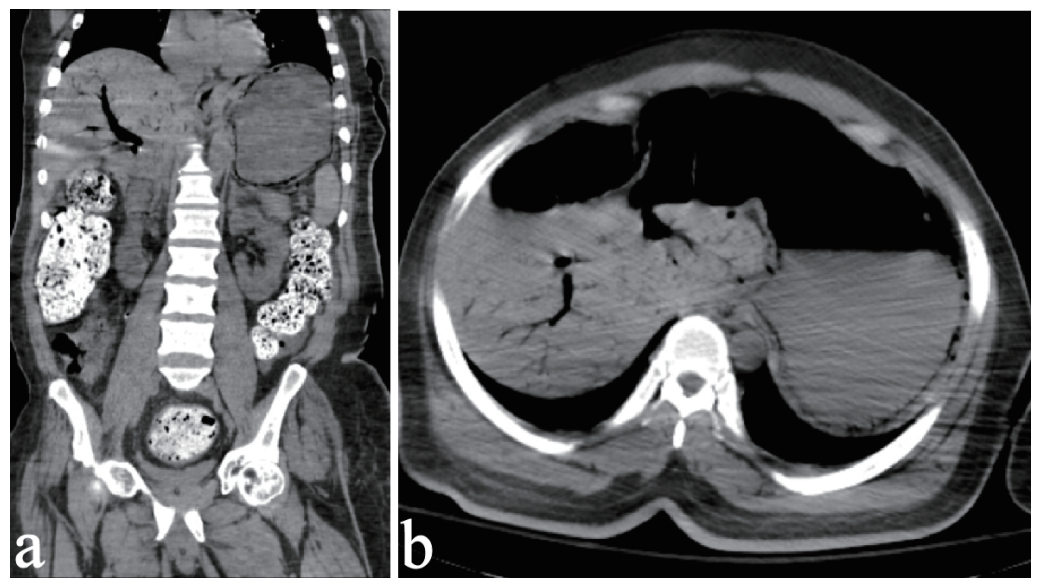

Figure 2. Right upper quadrant (RUQ) intrahepatic portal venous gas in frontal (a) section and transverse (b) section. 


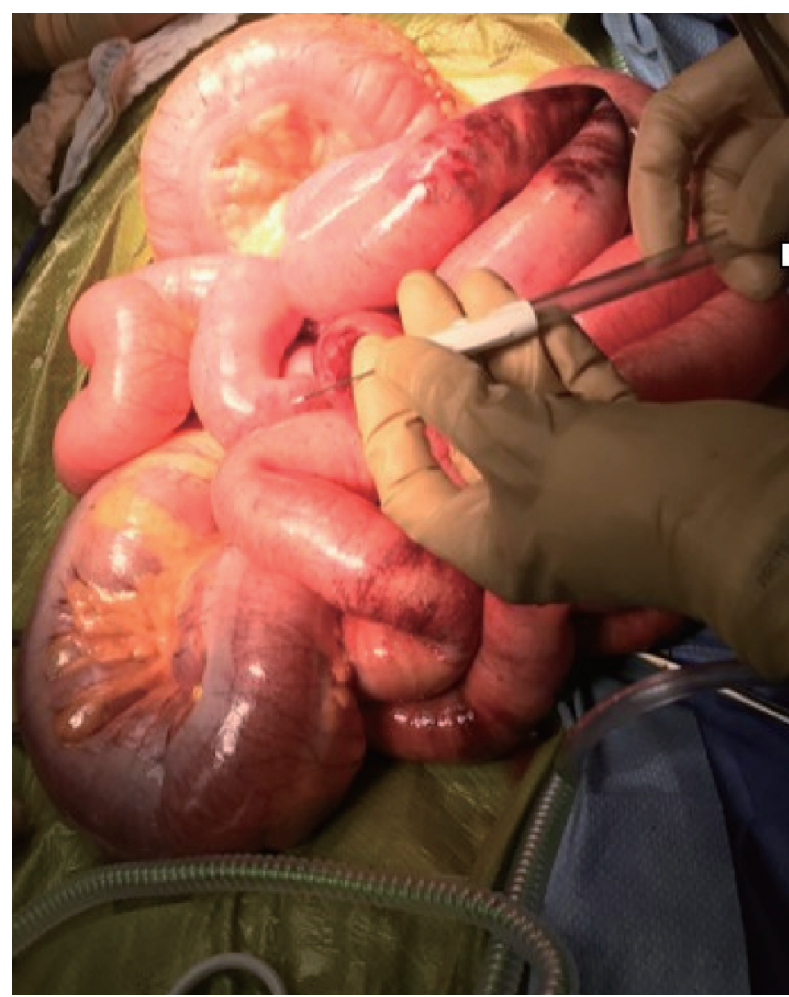

Figure 3. PI seen through small and large bowel with areas of ischemia. PI: pneumatosis intestinalis.

characterized by the inability of the body to maintain certain nutritional balances (protein, fluids, and electrolytes) that is a direct result of the surgical resection (or congenital) of the small bowel, usually when $200 \mathrm{~cm}$ or less of small bowel remaining. Early aggressive TPN should be performed with progressive weaning off and subsequent administration of enteral nutrition. Medications such as opiates and teduglutide, a glucagon-like peptide 2 agonist, may be administered to decrease stool output and improve intestinal growth/absorption, respectively.

Patients presenting with abdominal distension and history of constipation/decrease in frequency in bowel movements should have lactate levels checked and a sepsis workup administered, especially in a bedridden patient. The decrease in frequency of bowel movements may be as slight as 1 or 2 days. The presentation of PI and/or PVG is widely variable, which is why a non-contrast CT of the abdomen/pelvis is warranted in determining diagnosis and potential etiologies to patient presentation. Ultimately, any symptomatic patient with radiological evidence of PI and/or PVG should be hospitalized and started on an antibiotic treatment regimen. Determining the etiology, level of damage and therapeutic options available are crucial in treatment and recovery of PI and/or PVG, and may or may not justify exploratory laparotomy.

\section{Acknowledgments}

None to declare.

\section{Financial Disclosure}

There are no disclosures of relevant financial relationships or funding of any kind.

\section{Conflict of Interest}

There are no conflicts of interest in this study.

\section{Informed Consent}

Informed consent was obtained from the patient about using photographs and medical information for medical education but without using any identifying patient information.

\section{Author Contributions}

Acquisition of date: A. Zeinoun and C. Persaud; analysis and interpretation of data: A. Zeinoun, C. Persaud, and J. Zuberi; drafting of manuscript: A. Zeinoun and C. Persaud; critical revision: A. Zeinoun, C. Persaud, and J. Zuberi.

\section{References}

1. Wayne E, Ough M, Wu A, Liao J, Andresen KJ, Kuehn D, Wilkinson N. Management algorithm for pneumatosis intestinalis and portal venous gas: treatment and outcome of 88 consecutive cases. J Gastrointest Surg. 2010;14(3):437-448.

2. Kanwal D, Attia KME, Fam MNA, Khalil SMF, Alblooshi AM. Stercoral perforation of the rectum with faecal peritonitis and pneumatosis coli: A case Report. J Radiol Case Rep. 2017;11(3):1-6.

3. Chaput U, Ducrotte P, Denis P, Nouveau J. Pneumatosis cystoides intestinalis: an unusual cause of distal constipation. Gastroenterol Clin Biol. 2010;34(8-9):502-504. 